\title{
Developmental Plasticity in Plants
}

\author{
M. DE JONG AND O. LeYSER \\ Sainsbury Laboratory, Cambridge University, Cambridge CB2 1LR, United Kingdom \\ Correspondence: ol235@cam.ac.uk
}

\begin{abstract}
As sessile organisms, plants are unable to seek out environmental conditions optimal for their growth and development but instead must complete their life cycles in the environment in which they are growing. However, plants are remarkably plastic, such that a single genotype is able to give rise to a wide range of phenotypes. Developmental plasticity has profound implications for plant evolution and ecology and can make important contributions to improving yield stability in agriculture. In this review, we discuss the genetic control mechanisms that underlie plasticity and their implications for plant evolution, using the control of flowering time in Arabidopsis as an example. Furthermore, we consider how rapid improvements in quantitative genetic resources provide opportunities to analyze the molecular mechanisms that regulate developmental plasticity more directly and completely.
\end{abstract}

Higher plants are complex multicellular organisms that are autotrophic. They depend on the collection of relatively simple molecules from the environment-water, carbon dioxide, and mineral nutrients - and use energy from sunlight to power the conversion of these simple molecules into the complex macromolecules from which they are built. To collect sufficient quantities of these basic resources, plants must have very large surface areas in contact with both the above- and below-ground environments. As a result, they are sessile, unable to seek out environmental conditions optimal for their successful growth and development. Instead, wherever a seed germinates, the emerging plant must complete its life cycle at that location, whatever the environment. Furthermore, the environment is likely to change dynamically during the lifetime of the plant. Given these constraints, it is not surprising that plant development is remarkably plastic, such that a single plant genotype is able to give rise to a wide range of phenotypes depending on the prevailing environmental conditions (Bradshaw 1965; Sultan 2000).

This plasticity in development is possible because of the continuous nature of plant development (Palmer et al. 2012). During embryogenesis, the basic body axes of the plant are established, including the main apical-basal axis with the shoot apical meristem (SAM) at one end and the root apical meristem at the other. After germination, this axis is elaborated by the development of root and shoot systems, built through the action of the primary embryonic meristems, as well as the establishment of new secondary meristems, giving rise to lateral roots and shoots and higher-order branches. Each root and shoot can branch to different degrees, elongate to different degrees, and, particularly the shoot, produce a range of different specialized structures of varying types, to various degrees. For example, shoots can produce leaves adapted to shade or bright sunlight and, at some point, switch from making leaves to making floral organs. By integrating environmental information into the regulation of these growth and developmental processes, plant form can be modulated according to the environment in which the plant is growing. In this way, the final morphology of the plant depends on the environment, defining it as plastic.

The extraordinary phenotypic plasticity of plants has received attention in two interlinked contexts. First, developmental plasticity has profound implications for plant evolution. If a single genotype can result in multiple phenotypes, this weakens the link between selection at the level of the phenotype and changes in allele frequency at the level of the genotype. This suggests an explanation for why there are so many fewer plant species than animal species on the planet. However, it also raises interesting questions about the constraints on plasticity and the limits to the selective advantages that it brings, begging the question, under what circumstances is plasticity selected against? Second, it is becoming increasingly possible to investigate the genetic control mechanisms underlying plasticity. A priority in this context is the question of the relationship between plasticity in a trait and the mean value of that trait. As mentioned above, these two areas are interlinked, because understanding the genetic architecture of plasticity will contribute to understanding its evolution. In this review, we discuss progress toward this goal and the opportunities presented by spectacular improvements in quantitative genetic resources, enabled by cheap and easy DNA sequencing.

\section{THE EVOLUTIONARY SIGNIFICANCE OF PLASTICITY}

\section{Nonadaptive Plasticity}

As described above, the ability of plants to modulate their development in response to the environment is usually considered to be adaptive, contributing positively to reproductive success. However, before considering the implications for adaptive developmental plasticity, it is 
important to point out that phenotypic variation between plants grown in different environments may not affect fitness at all, or it may have a negative impact on fitness. Such variation may, for example, reflect variation in a trait as a result of mutation or selection of other traits that are linked (Alpert and Simms 2002), or it may reflect poorly buffered development with negative effects on individual fitness as the inevitable result of stress or resource limitation (Sultan 2000; Steinger et al. 2003; Weiner 2004; Van Kleunen and Fischer 2005).

Although adaptive plasticity is generally considered to be of most evolutionary and ecological interest, all these types of plasticity influence a plant's chances of survival and reproduction and will thus affect processes such as colonization. Nonadaptive plasticity reduces the likelihood of persistence in a new environment that is stressful and thus increases the strength of selection. Grether (2005) suggests that when a species encounters novel environmental conditions that trigger phenotypic changes with reduced fitness, selection will favor genetic changes that counteract these changes to restore the phenotype to its ancestral state. When this form of adaptive evolution, termed "genetic compensation," has occurred, genetic differences among populations have evolved so that the mean trait values of populations in different environments may appear to be more similar when measured in their native habitat than when grown under common environmental conditions. Thus, genetic compensation reduces phenotypic variation but promotes genetic divergence (Ghalambor et al. 2007; Fitzpatrick 2012).

Alternatively, stressful environments may increase phenotypic variation via the expression of cryptic genetic variation. Under typical environmental conditions, the observed phenotypic diversity is only a fraction of the diversity that is actually available, but under stressful conditions, cryptic genetic variation may be revealed. By chance, this stress-induced, nonadaptive plasticity could produce a phenotype that supports survival and reproduction, thereby allowing further adaptation to the new environmental conditions (Rutherford 2000; Gibson and Dworkin 2004; Ghalambor et al. 2007).

\section{Adaptive Plasticity}

The ability to modulate development according to the environment can clearly be adaptive by maintaining reproductive success in a changing environment. This is true both for a single individual growing in fluctuating environment and for populations growing in heterogeneous environments. These properties mean that phenotypic plasticity has important roles in the evolutionary diversification of plants (Via and Lande 1985; Schlichting 1986; Scheiner 1993) and in the distribution and persistence of plant species, for example, in response to global climate change (Reusch and Wood 2007; Chevin et al. 2010; Nicotra et al. 2010; Palmer et al. 2012). Populations with high plasticity in selectively important traits can rapidly adapt phenotypically to changes in environmental conditions without the need for genetic adaptation
(Valladares et al. 2007; Crispo 2008). Similarly, successful invasive species often show higher plasticity than the native species with which they are competing, allowing them to adjust rapidly to their new geographical area (Sultan 2003; Lande 2009).

The ability of a single genotype to compete successfully in diverse environments can result in reduced genetic variation at the population level, which can inhibit trait evolution (Schlichting 1986; Sultan 2000). If developmental plasticity results in adaptive phenotypes that match the optimum for relevant traits in a range of environments, there is no opportunity for directional selection and thus no genetic differentiation among populations across the environmental range (Ghalambor et al. 2007). Alternatively, if plasticity allows a population to persist in a changed or new environment, but does not deliver the optimal phenotype, the phenotype may become genetically fixed by genetic assimilation (Pigliucci et al. 2006) and improved further by natural selection. This phenomenon, also known as the "Baldwin effect" (Simpson 1953), can accelerate phenotypic adaptation to new growth conditions by allowing an entire population to persist by adaptive plasticity, with successful phenotypes honed by natural selection (Crispo 2008; Lande 2009).

\section{Costs and Limits}

Given its obvious benefits, the observation that there is substantial genetic variation in plasticity among natural plant populations suggests that it must also have costs (Pigliucci and Schlichting 1995; Linhart and Grant 1996; Van Kleunen and Fischer 2005). According to DeWitt et al. (1998), costs are evident when, under similar environmental conditions, the fitness of a plastic organism is lower compared with the fitness of a nonplastic organism with the same mean trait value. Several costs for plasticity have been identified (DeWitt et al. 1998; Auld et al. 2010). The most straightforward are those associated with sensing the environmental conditions and transducing these signals into developmental responses. More difficult to assess are costs that could result from the additional genetic complexity that may be required for plasticity. This could result in genetic costs from linkage between loci that promote plasticity and loci conferring low fitness. There could also be pleiotropic effects of loci affecting plasticity on other traits and epistatic interactions between plasticity-related loci and other loci. All of these could reduce the fitness of plastic organisms in comparison with those that have more fixed developmental programs.

In addition to these costs, plasticity clearly has limits that can also reduce fitness. This can be the case where there are developmental range limits such that the developmental outcome of a plastic response cannot produce trait values as near to the optimum as a more constrained, fixed developmental program. For example, in extreme environments, the optimum for a trait may be very far from the population mean and not attainable by an organism that can adopt a range of trait values around the mean. 
Because of this, phenotypic plasticity is often associated with lifestyle. Generalists living in moderate environments, with strong competition, often display a high degree of plasticity, whereas specialists that are restricted to harsher environmental conditions display a low degree of plasticity (Sultan 2003).

As well as developmental range limits, plasticity can be limited by information reliability. This occurs when the cues used to assess the environment are insufficiently reliable or specific to provide an accurate assessment or when the environmental conditions are so variable that the information available at any one time gives little indication of future conditions. This latter case is closely related to so-called lag-time limits. Here, the amount of time between sensing and responding to environmental cues results in a temporary mismatch between environment and phenotype (Van Kleunen and Fischer 2005; Valladares et al. 2007). This is particularly problematic if the environment changes at a rate faster than the lag time, such that these mismatches persist. Furthermore, unlike biochemical or physiological plasticity, where changes are generally reversible, developmental modifications are often irreversible (Pigliucci et al. 2006) and therefore represent a more significant risk. If the environment reverts, it may not be possible to adopt the previous developmental state, or it may preclude the adoption of further alternative states, should the environment change again. Thus, the potential for phenotypic plasticity is limited by the history of plastic responses of the plant (Weinig and Delph 2001).

\section{When Is Adaptive Plasticity a Selective Advantage?}

Because of these limits and costs to plasticity, it is clear that selection will act on plasticity as well as on mean trait value, and thus the plasticity of a trait should itself be considered a quantitative trait (Bradshaw 1965; Alpert and Simms 2002; Nicotra et al. 2010). Because of selection, the degree or pattern of plasticity varies among species, populations, and genotypes. Furthermore, plasticity can vary within a single genotype, depending for example on the environmental conditions or developmental stage of the individual (Schlichting 1986; Sultan 2000; Alpert and Simms 2002), so that plasticity is itself a plastic trait (Sultan 2000; Huber et al. 2012).

Natural selection will favor locally adapted plants when the environment is constant, whereas phenotypic plasticity may be more beneficial in spatially or temporally heterogeneous environments (Givnish 2002). Whether natural selection actually favors plasticity depends on the scale and amplitude of spatial heterogeneity and the duration of the environmental variation. Plasticity may be most advantageous if the environment varies at a scale between the size of the responding part of the plant (e.g., the leaf in the case of sun vs. shade leaves or even the whole plant for root vs. shoot balance) and the dispersal range of the offspring (Alpert and Simms 2002). Recently, Baythavong (2011) has been able to support this hypothesis with empirical data by characterizing the plant communities associated with serpentine and nonserpentine soils. Serpentine soils, derived from ultramafic rocks, exhibit low levels of essential macronutrients, low calcium-magnesium ratios, and toxic concentrations of several elements (Branco and Ree 2010). Sites containing patches of serpentine soil represent a significantly finer grained habitat than sites without serpentine patches. Plasticity in plant populations growing on a site containing patches of serpentine soil was found to be higher than in populations growing on sites with uniformly nonserpentine soils (Baythavong 2011).

Plasticity may also be most beneficial if the environmental variation matches the developmental range of the plastic genotype and if the amount of time needed to express the plastic response is shorter than the duration of the environmental state (Padilla and Adolph 1996; Alpert and Simms 2002). Because the effectiveness of the plastic response greatly depends on its timing, these environmental variations are even more likely to select for plasticity if they are predictable and thus preceded by environmental cues that accurately indicate future conditions (Alpert and Simms 2002).

Another important consideration is the complexity of the environment. A heterogeneous environment often varies with respect to multiple factors, such as light, temperature, humidity, and nutrient status, and the spatial and temporal scales may differ for each of these factors (Valladares et al. 2007). All of these different environmental cues must be detected and integrated to maximize individual plant fitness. This involves prioritization of phenotypic variation that addresses the most limiting environmental factor and resolution of conflicting inputs into the same output.

Despite the long history of both theoretical and empirical research on plasticity, the mechanisms that regulate developmental plasticity are still poorly understood. Understanding how environmental cues are integrated with endogenous developmental programs to produce a functional phenotype will provide more insight into the ecological and evolutionary implications of phenotypic plasticity and its evolution.

\section{THE MOLECULAR MECHANISMS OF DEVELOPMENTAL PLASTICITY}

As described above, in 1965 Bradshaw noted that there is considerable variation in plasticity, both within species and among closely related species, and proposed that the plasticity of a trait is under genetic control. The discussion over the nature of this genetic control has been somewhat theoretical and based around the idea that traits are determined by distinct sets of structural genes that contribute directly to the trait and regulatory genes that control the activity of the structural genes. The two main models proposed to account for the genetic control of plasticity involve different roles for structural versus regulatory genes (Schlichting and Pigliucci 1993; Via 1993).

In the first model, allelic sensitivity, structural genes are constitutively expressed, but the level of expression is 
directly affected by the environmental conditions. The sensitivity of each structural gene to the environment may vary, depending on the allele. In this model, direct selection on the trait value leads to a strongly correlated response of trait plasticity, and plasticity is achieved as a result of the combined environmental sensitivity of many structural genes, with no specific "plasticity genes" (Via 1993). In contrast, in the second model (regulatory control), the expression of structural genes is mediated by regulatory genes, for example, genes encoding transcription factors that control the level of expression of multiple structural genes. If the activity of these genes is under environmental control, such genes could act as "plasticity genes," exerting environmentally dependent control over the expression of structural genes, enabling the amount and pattern of the plastic response to evolve independently of the mean trait value (Schlichting 1986; Schlichting and Pigliucci 1993, 1995). Under this model, different regulatory genes can be expressed in different environments, enabling independent responses to different environmental inputs. This could contribute to the evolution of plasticity, because genes with environment-specific expression may experience relaxed selection and thus a higher relative mutation rate compared with constitutively expressed genes (Van Dyken and Wade 2010; Wund 2012). This type of regulatory architecture could also support the evolution of control mechanisms that anticipate environmental change, for example, through circadian gating (Schlichting and Pigliucci 1995; Givnish 2002).

Allelic sensitivity and regulatory control are obviously not mutually exclusive. Furthermore, they start with the notion that there are separable structural and regulatory genes, which is clearly not the case. Perhaps the most useful aspect of these models is in their predictions about "plasticity genes." In the allelic sensitivity model, there are no "plasticity genes," and variation in mean trait values and plasticity variation go hand in hand. In the regulatory control model, there are distinct plasticity genes, and changes in which can affect plasticity without affecting mean trait values. Exploration of these issues requires the molecular analysis of trait plasticity in an ecological context. This type of analysis is underway for several important and/or tractable traits, the most advanced of which is the environmental control of flowering, principally studied in Arabidopsis. The genetic architecture of flowering time plasticity and its implications for its adaptive significance are discussed below.

\section{PLASTICITY IN ARABIDOPSIS FLOWERING TIME}

The optimal time to flower for an annual plant such as Arabidopsis is a complex calculation. For Arabidopsis, flowering is a one-off irreversible decision that leads to the production of seed and the end of the life cycle. During the vegetative phase, shoot and root proliferation allows nutrient and photosynthate accumulation. The transition to flowering curtails this phase of resource accumulation, capping fecundity. The optimal time to spend in the vegetative phase to maximize fitness depends on the environment. This includes relatively predictable aspects of the environment caused by the season, such as day length and average temperature, and less predictable aspects, such as nutrient availability, salinity, or unseasonable temperature fluctuations. Monitoring reliable seasonal indicators such as day length provides information about whether the conditions for growth are likely to be favorable and get better or worse in the future. Under circumstances where a prolonged period of favorable conditions is anticipated, remaining vegetative, thereby accumulating resources, is likely to improve fitness. In contrast, indicators of less predictable, unfavorable conditions may promote early flowering, ensuring that at least some seeds are produced, or they may slow growth and delay flowering, freeing resources for protection from stressful conditions.

Initial efforts to understand flowering time focused on the mechanisms that integrate seasonal cues into the flowering program. Current understanding of these mechanisms has been summarized in excellent reviews (for example, see Mouradov et al. 2002; Putterill et al. 2004; Ausin et al. 2005; Corbesier and Coupland 2006; Michaels 2009; Amasino 2010; Jarillo and Pineiro 2011; Srikanth and Schmid 2011). Regulatory frameworks established through the study of the seasonal regulation of flowering are now being elaborated via more recent analyses of how less predictable environmental variables affect the system.

\section{Seasonal Regulation of Flowering}

Molecular genetic analysis of flowering time began with classical forward genetic approaches to identify genes involved in the major seasonal regulatory inputs into flowering, namely, day length and winter cold. These studies identified a core set of regulators that act as integrators for multiple inputs. The main floral integrators are SOC1 (SUPPRESSOR OF OVEREXPRESSION OF CONSTANS 1), FT (FLOWERING LOCUS T), and FD, and they in turn regulate the expression of floral meristem identity genes including APl (APETALA1) and $L F Y$ ( $L E A F Y)$ (Kobayashi et al. 1999; Abe et al. 2005; Wigge et al. 2005; Yoo et al. 2005) that drive the floral program. Arabidopsis is a facultative long-day plant; its flowering is accelerated in long days (16/8 h light/ dark) and delayed in short-day conditions $(8 / 16 \mathrm{~h}$ light/dark). Day length is detected by a so-called external coincidence mechanism involving the interaction of the circadian clock and light detection. In leaves, the clock protein GI (GIGANTEA) promotes the expression of $C O$ (CONSTANS) such that it peaks during the night in short-day conditions, but this occurs at dusk when plants are grown under long days (Suarez-Lopez et al. 2001). In darkness, the CO protein is rapidly degraded and thus never accumulates under short days. In long days, at the end of the day, light and CO coincide. The light is detected by the photoreceptors cry1 (cryptochrome 1) and cry2 and phyA (phytochrome A), resulting in stabilization of 
CO (Valverde et al. 2004). CO induces the expression of FT (Yanovsky and Kay 2002). FT encodes a small, soluble protein that moves through the phloem toward the SAM, where it interacts with the bZIP transcription factor FD to activate the floral meristem identity genes (Abe et al. 2005; Wigge et al. 2005).

In the meristem, independent of $\mathrm{CO}$, expression of the floral integrators $F T, S O C 1$, and $F D$ is regulated by FLC (FLOWERING LOCUS C), a member of the MADS box transcription factor family. FLC acts as a suppressor of flowering, and its expression is down-regulated during prolonged exposure to cold (vernalization) (Michaels and Amasino 1999). Arabidopsis accessions that live at more northerly latitudes have high levels of FLC expression driven by the FRI (FRIGIDA) gene (Clarke and Dean 1994). As a result, these accessions require a period of prolonged cold before they flower, defining the winter annual life history. In the short growing season available to these plants, natural selection apparently favors germination in the autumn, overwintering as a slow-growing vegetative plant, rapid establishment of faster growth as soon as the spring arrives, and flowering when the photoperiod reaches the required length. Down-regulation of $F L C$ by prolonged cold is maintained after vernalization by chromatin modification at FLC by a Polycomb repression complex (Doyle and Amasino 2009; Jiang et al. 2009). Interestingly, this repression is mitotically stable and functions as a memory of prior cold exposure, permitting rapid flowering at the appropriate photoperiod. FLC is also the main target of the so-called autonomous pathway that allows flowering eventually even in the absence of environmental conditions that accelerate flowering. Different autonomous pathway genes repress $F L C$ expression using different mechanisms. FCA and $F Y$ control FLC gene expression by regulating polyadenylation (Manzano et al. 2009). FLD (FLOWERING LOCUS D) and $F V E$ are involved in histone deacetylation and thus may repress FLC expression by changing chromatin structure (He et al. 2003; Ausin et al. 2004; Kim et al. 2004).

Recently, an additional endogenous pathway for flowering has been described involving two microRNAs, miR156 and miR172. In Arabidopsis, the expression of these miRNAs is independent of day length but is developmentally controlled. As development proceeds and the plant matures, miR156 levels decline, releasing the transcriptional repression of genes of the SPL (SQUAMOSA PROMOTER BINDING-LIKE) family (Wang et al. 2009). SPL transcription factors are positive regulators of floral integrators and may increase levels of miR172 (Wu et al. 2009; Yamaguchi et al. 2009). In turn, miR172 promotes flowering by down-regulating repressors of FT (Aukerman and Sakai 2003; Mathieu et al. 2009).

The plant growth regulator GA (gibberellin) also has an important role in the activation of flowering, which is especially evident under short-day conditions when the photoperiodic pathway is not activated. Under short days, GAs activate the expression of SOC1 and the floral meristem identity gene $L F Y$ (Blazquez et al. 1998; Moon et al. 2003). The activation of $L F Y$ by GA is possibly also mediated through miR159, which directs cleavage of mRNA encoding GAMYB-related transcription factors that regulate the activity of $L F Y$ (Gocal et al. 2001; Achard et al. 2004). Under long days, GA is required in the vascular tissue, where it increases the expression of FT and its closest homolog TSF (TWIN SISTER OF FT). In the meristem, GA activates the transcription of $S P L$ genes but is not required to activate $S O C 1$ as reported under short-day conditions (Hisamatsu and King 2008; Porri et al. 2012).

\section{Regulation of Flowering Time in Response to Nutrient Availability}

The inorganic nutrient nitrate $\left(\mathrm{NO}_{3}^{-}\right)$is a major source of nitrogen for plants. Low $\mathrm{NO}_{3}^{-}$does not statistically significantly reduce the number of days to flowering (Kant et al. 2011; Marín et al. 2011), but when flowering time is scored on the basis of leaf number, plants grown on low $\mathrm{NO}_{3}^{-}$flower earlier, suggesting that differences in flowering time are masked by differences in growth rate (Marín et al. 2011). Consistent with this view, in Arabidopsis, several studies have shown that the number of days to flowering is not significantly affected by mineral nutrition (Zhang and Lechowicz 1994; Pigliucci and Schlichting 1995). However, when plants germinated and grown on high nutrient levels for several weeks were transferred to low nutrient levels, flowering was promoted. This effect of nutrient deficiency was more pronounced in short days than in long days (Kolár and Senkova 2008).

In an alternative approach to reduce growth differences between treatments, Marín et al. (2011) studied the effect of varying concentrations of $\mathrm{NO}_{3}^{-}$on flowering while providing constant $\mathrm{N}$ nutrition through glutamine supply. Decreased $\mathrm{NO}_{3}^{-}$resulted in earlier flowering but only in short-day conditions, consistent with previous results (Kolár and Senkova 2008). Mutants and transgenic lines affecting the autonomous, GA, photoperiod, or vernalization pathways were still able to respond to nitrate. Strikingly, the triple mutant $f_{c a}-1$ co-2 gal-3 did not flower under either short- or long-day conditions but did flower when grown under low- $\mathrm{NO}_{3}^{-}$conditions. Other abiotic stresses such as high light or high temperature could not induce flowering in this triple mutant. Furthermore, low $\mathrm{NO}_{3}^{-}$also accelerated flowering in floral integrator mutants such as $f t$ and $f d$ and floral identity mutants such as lfy. These findings suggest that the response to nitrate is not part of a general stress response but is regulated by a separate pathway, parallel to the photoperiod, GA, and autonomous pathways, that enters downstream from known floral integrators (Fig. 1). Interestingly, transgenic lines that constitutively overexpressed FLC did not respond to nitrate, but plants grown on low $\mathrm{NO}_{3}^{-}$have reduced levels of FLC mRNA (Kant et al. 2011; Marín et al. 2011). Possibly, low nitrate represses FLC but only when it is expressed from its own promoter (Fig. 1). 


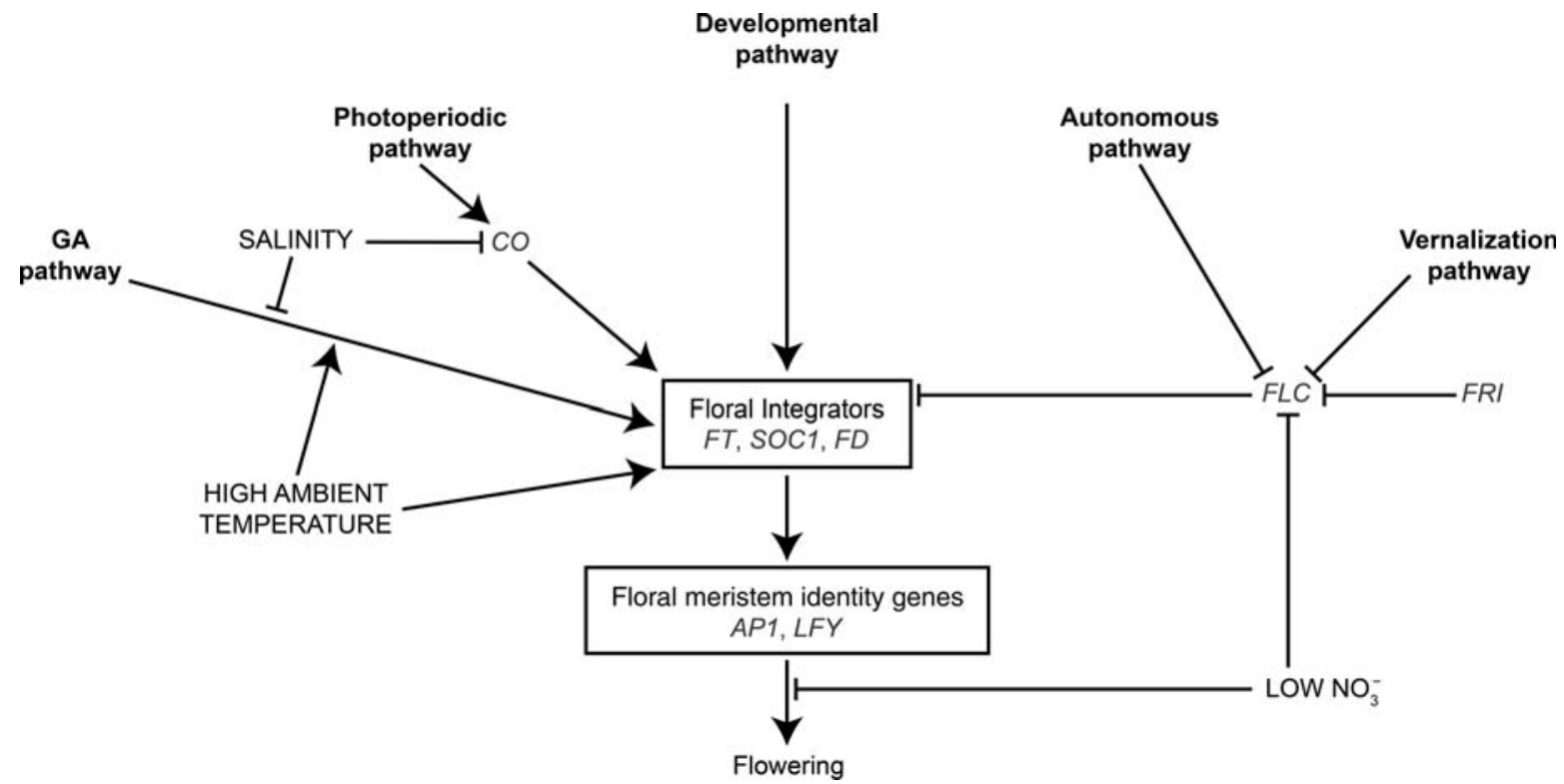

Figure 1. Schematic model of the pathways that control flowering time in Arabidopsis. Genetic studies have identified regulatory pathways (in bold) that integrate the seasonal environmental cues of day length and winter into the endogenous flowering program. Less predictable environmental cues, such as nutrient availability, salinity, or unseasonal temperature fluctuation, also affect flowering time. Most of these responses are regulated through the well-described pathways, modifying the activity of genes involved in the photoperiodic, autonomous, and GA floral pathways. Floral integrators, such as $F T, S O C 1$, and $F D$, act as hubs that integrate multiple inputs to regulate flowering by modulating the expression of floral meristem identity genes, such as $A P 1$ and $L F Y$. Some environmental cues, however, such as low nitrate $\left(\mathrm{NO}_{3}^{-}\right)$, may regulate flowering time independently of these pathways.

\section{Regulation of Flowering Time in Response to Ambient Temperature}

In Arabidopsis, exposure to high temperature results in extension of the plant axes and early flowering (Gray et al. 1998). These phenotypes are similar to those produced in response to shading (Franklin 2008). Interestingly, phyB, which has a prominent role in the perception of shading, is also involved in temperature-sensitive flowering. When grown under a constant temperature of $22^{\circ} \mathrm{C}$, the $p h y B$ mutant displays an early flowering phenotype compared with wild type. Accordingly, FT expression is greatly enhanced. However, the early flowering phenotype of the $p h y B$ mutant is abolished when grown at the lower temperature of $16^{\circ} \mathrm{C}$, and $F T$ transcript levels are reduced to wild-type levels at this temperature. Additional loss of $P H Y E$ next to $P H Y B$ accelerates flowering at $16^{\circ} \mathrm{C}$ and triggers an elevation in $F T$ transcription. These data support a role for FT in the ambient-temperaturedependent induction of flowering mediated by the phytochromes (Halliday et al. 2003). In the same study, the different ambient temperatures did not alter the expression of FLC transcript levels, suggesting that ambient temperature regulation of flowering is independent of the autonomous floral pathway (Fig. 1).

An increase in growth temperature from $23^{\circ} \mathrm{C}$ to $27^{\circ} \mathrm{C}$ can induce flowering, bypassing the requirement for long photoperiods, and can induce flowering in double mutants of $f l c$ upstream of autonomous pathway mutants. Single mutants of the autonomous pathway, however, were unable to respond to thermal induction. Natural accessions of Arabidopsis that have lesions at FLC or $F R I$ were more sensitive to temperature than the lines with apparently functional FRI/FLC alleles (Balasubramanian et al. 2006). These results suggest a role for FLC in suppressing thermal induction. Further exploitation of the natural variation in thermal induction also identified FLM (FLOWERING LOCUS M), a paralog of FLC, as a major-effect quantitative trait locus modulating thermosensitivity. Accessions lacking functional FLM respond less well to temperature than accessions with functional FLM, which likely signals through modulation of FT levels (Balasubramanian et al. 2006).

The up-regulation of FT expression in response to temperature is also mediated through the basic helix-loophelix (bHLH) transcriptional regulator PIF4 (PHYTOCHROME INTERACTING FACTOR 4) (Koini et al. 2009). Mutants with lesions in PIF4 showed a loss of thermal induction of flowering and abolished FT expression at high ambient temperature in short days, whereas overexpression of PIF4 caused early flowering. Kumar et al. (2012) showed that PIF4 directly binds to the FT promoter near the transcriptional start site and that temperature-dependent regulation of FT by PIF4 proteins is controlled at the level of chromatin accessibility. The protein activity of PIF4 is controlled by DELLA proteins; these are repressors of GA-responsive growth. DELLA proteins are able to bind to the DNA-recognition domain of PIF4 (de Lucas et al. 2008), preventing PIF4 from binding to the promoter of FT. The phytohormone GA induces degradation of DELLA proteins via the ubiquitin/proteasome pathway. Accordingly, GA application increased the 
expression of FT (Hisamatsu and King 2008). These findings suggest that PIF4 acts as an integrator between the ambient-temperature-sensing pathway and the GA floral induction pathway (Kumar et al. 2012) (Fig. 1).

\section{Regulation of Flowering Time in Response to Salinity}

In contrast to the previously discussed environmental cues, salt stress delays flowering in Arabidopsis. Flowering in response to salt is regulated in a dose-dependent manner. Moderate salt stress promotes vegetative growth, but delays flowering, whereas high concentrations of salt repress both vegetative and reproductive growth. Salttreated wild-type plants contain reduced levels of bioactive GAs and substantially reduced expression of $L F Y$ (Achard et al. 2006). Exogenous GA application can partially rescue both the late-flowering phenotype and $L F Y$ expression levels of salt-treated plants, suggesting that the regulation of flowering time by salt is partly mediated by the GA pathway. Mutants with lesions in FLC or FCA, however, are still responsive to salinity, suggesting that the autonomous pathway is not involved in salt-induced repression of floral transition (Fig. 1).

$C O$ and $F T$ mRNA levels are reduced by salt stress in a dose-dependent manner and the co-2 mutant is not responsive to moderate salt stress (Fig. 1) (Li et al. 2007). An additional mechanism to regulate $F T$ expression in response to salinity involves the transcription factor NTM1-LIKE8 (NTL8), a member of the NAM, ATAF1/ 2, CUC2 (NAC) transcription factor family. The expression of NTL8 is induced by high salt. The NTL8 protein is primarily localized at the plasma membrane, but if released, NTL8 translocates to the nucleus to regulate the expression of target genes. Overexpressing nuclear NTL8 delays flowering and is associated with the reduced expression of $F T$. In the $n t l 8-1$ knockout mutant, however, the salt-induced repression of FT is diminished (Kim et al. 2007). In contrast to $F T$, its gene family member $B F T$ (BROTHER OF FT AND TFL1) is induced under high salinity, whereas other environmental factors, such as cold and drought, only slightly affect its expression. The salt induction of BFT expression is largely mediated by the stress-related phytohormone ABA (abscisic acid). Overexpression of BFT delayed flowering under both normal growth conditions and high salinity, whereas the $b f t-2 \mathrm{mu}-$ tant was insensitive to high salinity. BFT regulates the meristem identity genes, such as $L F Y$ and $A P 1$, but only $A P 1$ appears to be involved in BFT regulation of flowering under high salinity (Ryu et al. 2011).

\section{Central Regulation of Flowering Time and Flowering Time Plasticity}

As is clear from the evidence presented above, different environmental cues accelerate or delay the transition to flowering. The genes FLC and FT, which are the central players in the well-described seasonal flowering time pathways, are also key components in the regulation of flowering in response to less predictable environmental cues. Indeed, most of these environmentally induced responses are regulated through the photoperiodic, autonomous, or GA floral pathways (Fig. 1). Nevertheless, some environmental cues may regulate flowering time independently of these pathways, bypassing known floral integrators and identify genes. Another recurring feature is that these environmental inputs frequently influence flowering by multiple signaling routes, resulting in both redundancy and complexity in the mechanisms that control flowering.

These features have interesting hybrid properties between the allelic sensitivity and regulatory control models. On the one hand, there are clearly "plasticity genes." For example, FRI functions to set the level of FLC, thereby determining the extent to which a plant requires vernalization to flower. If vernalized, FRI and fri plants flower at the same time, but without vernalization, their flowering times are dramatically different. This defines FRI as a "plasticity gene." Furthermore, there is clearly a regulatory hierarchy allowing multiple different environmental inputs to be integrated by an overlapping set of common targets to regulate a single output, characteristic of the regulatory control model. However, the redundancy in the system, with one environmental signal targeting multiple genes in the regulatory network, is reminiscent of the allelic sensitivity idea, and some environmental inputs, such as nitrate, have substantial effects on flowering time through as yet unknown mechanisms that apparently feed in downstream from known regulators, closer to the "structural genes."

\section{Natural Variation in Flowering Time Plasticity}

All of these studies, although building a detailed network for the control of the floral transition, were not really intended to specifically address the question of plasticity. The mutant screens that were the mainstay of the identification of the genes described involved selection for mean trait value in a single environment. Many different environments have been used in different screens, but nonetheless, this approach does not screen directly for plasticity per se. This requires individuals with identical genotypes to be grown in two or more environments. Therefore, the use of classical mutant screens to identify candidate genes involved in phenotypic plasticity is challenging. In a typical mutant screen, single M2 plants are selected based on phenotype, but to identify plasticity mutants, M3 families would have to be collected and scored in two relevant environmental conditions. Moreover, plasticity is difficult to study using null alleles, which are the most common type recovered from mutant screens. Null alleles simultaneously remove the function of the gene and any ability to modulate it, making it difficult to separate mean trait value from plasticity in the trait. These problems can be overcome through the use of natural quantitative variation mapped in recombinant inbred lines using traditional linkage mapping or genome-wide association (GWA) mapping to identify quantitative trait loci (QTL). Both molecular tools are based on the statistical association 
of phenotypic data with genotypic data (Ikram and Chardon 2010).

Traditional linkage mapping in recombinant inbred lines (RILs) is well suited to the identification of genomic regions governing plasticity. RILs are almost completely homozygous, allowing replicates to be grown and analyzed across several environments. Mapping resolution and genetic diversity in the mapping population is determined by the number of founder lines and the generations of intermating and selfing used to make the RILs (Bergelson and Roux 2010). For example, Kover et al. (2009b) developed a set of RILs derived from a multiparent advanced generation intercross (MAGIC) of 19 accessions of Arabidopsis. The mapping resolution for the MAGIC lines can be as high as $300 \mathrm{~kb}$, so these lines can be used for fine-mapping QTL including phenotypic plasticity.

In natural accessions, recombination events have accumulated over many generations, so the mapping resolution in GWA studies can be higher than in traditional mapping. Self-fertilizing species such as Arabidopsis are well suited for GWA studies. Once the accessions have been densely genotyped, they can be used to analyze different traits among different environments (Nordborg and Weigel 2008). These approaches have now been quite extensively used to map variation in flowering time, including studies carried out on the same lines in different conditions (see, e.g., Schwartz et al. 2009; Brachi et al. 2010; Li et al. 2010; Strange et al. 2011). Most of the subsequent molecular analysis has focused on known loci, already identified in classical mutant screens. Although this has revealed important data on the molecular basis for natural variation in flowering time, it has been less relevant from the point of view of analysis of phenotypic plasticity. This may change as the focus shifts to previously uncharacterised loci or studies of plastic responses to nonseasonal inputs. Similarly, analysis of traits other than flowering time that can be modulated more continuously is now under way (see, e.g., Filiault and Maloof 2012). For these inputs and traits, there is likely to be less straightforward selection on plasticity, so the genetic architecture of plasticity in these traits may be more revealing.

A further use of these lines that can, for example, help to distinguish between allelic sensitivity and regulatory control models is through selection experiments (Scheiner 2002). These are challenging in higher plants owing to their relatively long generation times. Nevertheless, several artificial selection studies have been conducted with Nicotiana rustica and Arabidopsis to evaluate the independence between mean trait value and trait plasticity. These have variously targeted the mean trait values, the plasticity of the trait, or a combination of the two for selection (Brumpton et al. 1977; Jinks et al. 1977; Callahan and Pigliucci 2005; Springate et al. 2011). The findings of these studies suggest that phenotypic plasticity is at least partially under separate genetic control from the trait value, supporting the regulatory control hypothesis. For example, in an artificial selection experiment carried out by Kover et al. (2009a), outbred lines of Arabidopsis were selected for early flowering under either winter-annual or spring-annual simulated conditions. At the end of the experiment, the selected lines always flowered earlier than the control lines, independent of the growth conditions. However, when grown in the conditions in which they were selected, the lines always flowered earlier than the lines selected in the alternative environment. These findings suggest that some of the selected genes affect flowering time under both growing conditions, whereas others are involved specifically in the environmental control of flowering and therefore could be described as "plasticity genes." Molecular analysis suggests that one of these is FRI (Scarcelli and Kover 2009).

\section{Conclusion and Prospects}

Developmental plasticity has shaped the evolutionary history of plants so that understanding the genetic architecture of plasticity is a prerequisite for understanding plant evolution and ecology. Rapid progress has been achieved through advances in plant developmental genetics, with opportunities for the future provided by postgenomic quantitative genetics. These advances are timely because understanding plasticity is also paramount for progress in crop science. Phenotypic plasticity is an important trait for the yield stability of crops. Crops have typically been bred for uniform growth and higher yields under local conditions and often have been selected for reduced genotype-by-environment interactions (Palmer et al. 2012). Thus far, directional selection has not focused on phenotypic plasticity (Nicotra et al. 2010), despite the fact that yield stability and quality in crops such as cereals and rapeseed is often compromised by fluctuating weather conditions (Peltonen-Sainio et al. 2011). Therefore, selective breeding aimed at the plasticity of traits indirectly and directly associated with yield may allow the development of crops with improved yield stability in an increasingly unpredictable environment.

\section{REFERENCES}

Abe M, Kobayashi Y, Yamamoto S, Daimon Y, Yamaguchi A, Ikeda Y, Ichinoki H, Notaguchi M, Goto K, Araki T. 2005. FD, a bZIP protein mediating signals from the floral pathway integrator FT at the shoot apex. Science 309: 1052-1056.

Achard P, Herr A, Baulcombe DC, Harberd NP. 2004. Modulation of floral development by a gibberellin-regulated microRNA. Development 131: 3357-3365.

Achard P, Cheng H, De Grauwe L, Decat J, Schoutteten H, Moritz T, Van Der Straeten D, Peng J, Harberd NP. 2006. Integration of plant responses to environmentally activated phytohormonal signals. Science 311: 91-94.

Alpert P, Simms EL. 2002. The relative advantages of plasticity and fixity in different environments: When is it good for a plant to adjust? Evol Ecol 16: 285-297.

Amasino R. 2010. Seasonal and developmental timing of flowering. Plant J 61: 1001-1013.

Aukerman MJ, Sakai H. 2003. Regulation of flowering time and floral organ identity by a MicroRNA and its APETALA2-like target genes. Plant Cell 15: 2730-2741.

Auld JR, Agrawal AA, Relyea RA. 2010. Re-evaluating the costs and limits of adaptive phenotypic plasticity. Proc $R$ Soc Lond, Ser B: Biol Sci 277: 503-511. 
Ausin I, Alonso-Blanco C, Jarillo JA, Ruiz-Garcia L, MartinezZapater JM. 2004. Regulation of flowering time by FVE, a retinoblastoma-associated protein. Nat Genet 36: 162-166.

Ausin I, Alonso-Blanco C, Martinez-Zapater JM. 2005. Environmental regulation of flowering. Int J Dev Biol 49: 689705.

Balasubramanian S, Sureshkumar S, Lempe J, Weigel D. 2006. Potent induction of Arabidopsis thaliana flowering by elevated growth temperature. PLoS Genet 2: e106.

Baythavong BS. 2011. Linking the spatial scale of environmental variation and the evolution of phenotypic plasticity: Selection favors adaptive plasticity in fine-grained environments. Am Nat 178: 75-87.

Bergelson J, Roux F. 2010. Towards identifying genes underlying ecologically relevant traits in Arabidopsis thaliana. Nat Rev Genet 11: 867-879.

Blazquez MA, Green R, Nilsson O, Sussman MR, Weigel D. 1998. Gibberellins promote flowering of Arabidopsis by activating the $L E A F Y$ promoter. Plant Cell 10: 791-800.

Brachi B, Faure N, Horton M, Flahauw E, Vazquez A, Nordborg M, Bergelson J, Cuguen J, Roux F. 2010. Linkage and association mapping of Arabidopsis thaliana flowering time in nature. PLoS Genet 6: e1000940.

Bradshaw AD. 1965. Evolutionary significance of phenotypic plasticity in plants. Adv Genet 13: $115-155$.

Branco S, Ree RH. 2010. Serpentine soils do not limit mycorrhizal fungal diversity. PLoS One 5: e11757.

Brumpton RJ, Boughey H, Jinks JL. 1977. Joint selection for both extremes of mean performance and of sensitivity to a macro-environmental variable. Heredity 38: 219-226.

Callahan HS, Pigliucci M. 2005. Indirect consequences of artificial selection on plasticity to light quality in Arabidopsis thaliana. J Evol Biol 18: 1403-1415.

Chevin L-M, Lande R, Mace GM. 2010. Adaptation, plasticity, and extinction in a changing environment: Towards a predictive theory. PLoS Biol 8: e1000357.

Clarke JH, Dean C. 1994. Mapping FRI, a locus controlling flowering time and vernalization response in Arabidopsis thaliana. Mol Gen Genet 242: 81-89.

Corbesier L, Coupland G. 2006. The quest for florigen: A review of recent progress. J Exp Bot 57: 3395-3403.

Crispo E. 2008. Modifying effects of phenotypic plasticity on interactions among natural selection, adaptation and gene flow. J Evol Biol 21: 1460-1469.

de Lucas M, Daviere JM, Rodriguez-Falcon M, Pontin M, Iglesias-Pedraz JM, Lorrain S, Fankhauser C, Blazquez MA, Titarenko E, Prat S. 2008. A molecular framework for light and gibberellin control of cell elongation. Nature 451: 480484.

DeWitt TJ, Sih A, Wilson DS. 1998. Costs and limits of phenotypic plasticity. Trends Ecol Evol 13: 77-81.

Doyle MR, Amasino RM. 2009. A single amino acid change in the enhancer of zeste ortholog CURLY LEAF results in vernalization-independent, rapid flowering in Arabidopsis. Plant Physiol 151: 1688-1697.

Filiault DL, Maloof JN. 2012. A genome-wide association study identifies variants underlying the Arabidopsis thaliana shade avoidance response. PLoS Genet 8: e1002589.

Fitzpatrick BM. 2012. Underappreciated consequences of phenotypic plasticity for ecological speciation. Int $J$ Ecol 2012: $1-12$.

Franklin KA. 2008. Shade avoidance. New Phytol 179: 930944.

Ghalambor CK, McKay JK, Carroll S, Reznick DN. 2007. Adaptive versus non-adaptive phenotypic plasticity and the potential for contemporary adaptation to new environments. Funct Ecol 21: 394-407.

Gibson G, Dworkin I. 2004. Uncovering cryptic genetic variation. Nat Rev Genet 5: 681-690.

Givnish TJ. 2002. Ecological constraints on the evolution of plasticity in plants. Evol Ecol 16: 213-242.

Gocal GF, Sheldon CC, Gubler F, Moritz T, Bagnall DJ, MacMillan CP, Li SF, Parish RW, Dennis ES, Weigel D, et al.
2001. GAMYB-like genes, flowering, and gibberellin signaling in Arabidopsis. Plant Physiol 127: 1682-1693.

Gray WM, Ostin A, Sandberg G, Romano CP, Estelle M. 1998. High temperature promotes auxin-mediated hypocotyl elongation in Arabidopsis. Proc Natl Acad Sci 95: 7197-7202.

Grether Gregory F. 2005. Environmental change, phenotypic plasticity, and genetic compensation. Am Nat 166: E115E123.

Halliday KJ, Salter MG, Thingnaes E, Whitelam GC. 2003. Phytochrome control of flowering is temperature sensitive and correlates with expression of the floral integrator FT. Plant J 33: 875-885.

He Y, Michaels SD, Amasino RM. 2003. Regulation of flowering time by histone acetylation in Arabidopsis. Science 302: $1751-1754$.

Hisamatsu T, King RW. 2008. The nature of floral signals in Arabidopsis. II. Roles for FLOWERING LOCUS T (FT) and gibberellin. J Exp Bot 59: 3821-3829.

Huber H, Chen X, Hendriks M, Keijsers D, Voesenek L, Pierik R, Poorter H, de Kroon H, Visser EJW. 2012. Plasticity as a plastic response: How submergence-induced leaf elongation in Rumex palustris depends on light and nutrient availability in its early life stage. New Phytol 194: 572-582.

Ikram S, Chardon F. 2010. Plant quantitative traits. In Encyclopedia of Life Sciences. Wiley \& Sons, Ltd. Chichester, UK.

Jarillo JA, Pineiro M. 2011. Timing is everything in plant development: The central role of floral repressors. Plant Sci 181: $364-378$.

Jiang D, Gu X, He Y. 2009. Establishment of the winter-annual growth habit via FRIGIDA-mediated histone methylation at FLOWERING LOCUS C in Arabidopsis. Plant Cell 21: $1733-1746$.

Jinks JL, Jayasekara NEM, Boughey H. 1977. Joint selection for both extremes of mean performance and of sensitivity to a macroenvironmental variable. Heredity 39: 345-355.

Kant S, Peng M, Rothstein SJ. 2011. Genetic regulation by NLA and MicroRNA827 for maintaining nitrate-dependent phosphate homeostasis in Arabidopsis. PLoS Genet 7: e1002021.

Kim HJ, Hyun Y, Park JY, Park MJ, Park MK, Kim MD, Lee MH, Moon J, Lee I, Kim J. 2004. A genetic link between cold responses and flowering time through FVE in Arabidopsis thaliana. Nat Genet 36: 167-171.

Kim SG, Kim SY, Park CM. 2007. A membrane-associated NAC transcription factor regulates salt-responsive flowering via FLOWERING LOCUS T in Arabidopsis. Planta 226: 647-654.

Kobayashi Y, Kaya H, Goto K, Iwabuchi M, Araki T. 1999. A pair of related genes with antagonistic roles in mediating flowering signals. Science 286: 1960-1962.

Koini MA, Alvey L, Allen T, Tilley CA, Harberd NP, Whitelam GC, Franklin KA. 2009. High temperature-mediated adaptations in plant architecture require the bHLH transcription factor PIF4. Curr Biol 19: 408-413.

Kolár J, Senkova J. 2008. Reduction of mineral nutrient availability accelerates flowering of Arabidopsis thaliana. J Plant Physiol 165: 1601-1609.

Kover PX, Rowntree JK, Scarcelli N, Savriama Y, Eldridge T, Schaal BA. 2009a. Pleiotropic effects of environment-specific adaptation in Arabidopsis thaliana. New Phytol 183: $816-825$

Kover PX, Valdar W, Trakalo J, Scarcelli N, Ehrenreich IM, Purugganan MD, Durrant C, Mott R. 2009b. A multiparent advanced generation inter-cross to fine-map quantitative traits in Arabidopsis thaliana. PLoS Genet 5: e1000551.

Kumar SV, Lucyshyn D, Jaeger KE, Alos E, Alvey E, Harberd NP, Wigge PA. 2012. Transcription factor PIF4 controls the thermosensory activation of flowering. Nature 484: 242-245.

Lande R. 2009. Adaptation to an extraordinary environment by evolution of phenotypic plasticity and genetic assimilation. $J$ Evol Biol 22: 1435-1446.

Li K, Wang Y, Han C, Zhang W, Jia H, Li X. 2007. GA signaling and $C O / F T$ regulatory module mediate salt-induced late 
flowering in Arabidopsis thaliana. Plant Growth Regul 53: $195-206$.

Li Y, Huang Y, Bergelson J, Nordborg M, Borevitz JO. 2010. Association mapping of local climate-sensitive quantitative trait loci in Arabidopsis thaliana. Proc Natl Acad Sci 107: 21199-21204.

Linhart YB, Grant MC. 1996. Evolutionary significance of local genetic differentiation in plants. Annu Rev Ecol Syst 27: $237-$ 277.

Manzano D, Marquardt S, Jones AM, Baurle I, Liu F, Dean C. 2009. Altered interactions within FY/AtCPSF complexes required for Arabidopsis FCA-mediated chromatin silencing. Proc Natl Acad Sci 106: 8772-8777.

Marín IC, Loef I, Bartetzko L, Searle I, Coupland G, Stitt M, Osuna D. 2011. Nitrate regulates floral induction in Arabidopsis, acting independently of light, gibberellin and autonomous pathways. Planta 233: 539-552.

Mathieu J, Yant LJ, Murdter F, Kuttner F, Schmid M. 2009. Repression of flowering by the miR172 target SMZ. PLoS Biol 7: e1000148.

Michaels SD. 2009. Flowering time regulation produces much fruit. Curr Opin Plant Biol 12: 75-80.

Michaels SD, Amasino RM. 1999. FLOWERING LOCUS C encodes a novel MADS domain protein that acts as a repressor of flowering. Plant Cell 11: 949-956.

Moon J, Suh SS, Lee H, Choi KR, Hong CB, Paek NC, Kim SG, Lee I. 2003. The SOC1 MADS-box gene integrates vernalization and gibberellin signals for flowering in Arabidopsis. Plant J 35: 613-623.

Mouradov A, Cremer F, Coupland G. 2002. Control of flowering time. Plant Cell 14: S111-S130.

Nicotra AB, Atkin OK, Bonser SP, Davidson AM, Finnegan EJ, Mathesius U, Poot P, Purugganan MD, Richards CL, Valladares F, et al. 2010. Plant phenotypic plasticity in a changing climate. Trends Plant Sci 15: 684-692.

Nordborg M, Weigel D. 2008. Next-generation genetics in plants. Nature 456: 720-723.

Padilla DK, Adolph SC. 1996. Plastic inducible morphologies are not always adaptive: The importance of time delays in a stochastic environment. Evol Ecol 10: 105-117.

Palmer CM, Bush SM, Maloof JN. 2012. Phenotypic and developmental plasticity in plants. In Encyclopedia of Life Sciences. Wiley \& Sons, Ltd. Chichester, UK.

Peltonen-Sainio P, Jauhiainen L, Sadras VO. 2011. Phenotypic plasticity of yield and agronomic traits in cereals and rapeseed at high latitudes. Field Crops Res 124: 261-269.

Pigliucci M, Schlichting CD. 1995. Reaction norms of Arabidopsis (Brassicaceae). III. Response to nutrients in 26 populations from a worldwide collection. Am J Bot 82: 11171125.

Pigliucci M, Murren CJ, Schlichting CD. 2006. Phenotypic plasticity and evolution by genetic assimilation. J Exp Biol 209: $2362-2367$.

Porri A, Torti S, Romera-Branchat M, Coupland G. 2012. Spatially distinct regulatory roles for gibberellins in the promotion of flowering of Arabidopsis under long photoperiods. Development 139: 2198-2209.

Putterill J, Laurie R, Macknight R. 2004. It's time to flower: The genetic control of flowering time. Bioessays 26: 363-373.

Reusch TBH, Wood TE. 2007. Molecular ecology of global change. Mol Ecol 16: 3973-3992.

Rutherford SL. 2000. From genotype to phenotype: Buffering mechanisms and the storage of genetic information. Bioessays 22: $1095-1105$.

Ryu J, Park C-M, Seo P. 2011. The floral repressor BROTHER OF FT AND TFL1 (BFT) modulates flowering initiation under high salinity in Arabidopsis. Mol Cells 32: 295-303.

Scarcelli N, Kover PX. 2009. Standing genetic variation in FRIGIDA mediates experimental evolution of flowering time in Arabidopsis. Mol Ecol 18: 2039-2049.

Scheiner SM. 1993. Genetics and evolution of phenotypic plasticity. Annual Review of Ecology and Systematics 24: $35-68$.
Scheiner SM. 2002. Selection experiments and the study of phenotypic plasticity. J Evol Biol 15: 889-898.

Schlichting CD. 1986. The evolution of phenotypic plasticity in plants. Annu Rev Ecol Syst 17: 667-693.

Schlichting CD, Pigliucci M. 1993. Control of phenotypic plasticity via regulatory genes. Am Nat 142: 366-370.

Schlichting CD, Pigliucci M. 1995. Gene regulation, quantitative genetics and the evolution of reaction norms. Evol Ecol 9: $154-168$.

Schwartz C, Balasubramanian S, Warthmann N, Michael TP, Lempe J, Sureshkumar S, Kobayashi Y, Maloof JN, Borevitz JO, Chory J, et al. 2009. Cis-regulatory changes at FLOWERING LOCUS $\mathrm{T}$ mediate natural variation in flowering responses of Arabidopsis thaliana. Genetics 183: $723-$ 732.

Simpson GG. 1953. The Baldwin effect. Evolution 7: 110-117. Springate DA, Scarcelli N, Rowntree J, Kover PX. 2011. Correlated response in plasticity to selection for early flowering in Arabidopsis thaliana. J Evol Biol 24: 2280-2288.

Srikanth A, Schmid M. 2011. Regulation of flowering time: All roads lead to Rome. Cell Mol Life Sci 68: 2013-2037.

Steinger T, Roy BA, Stanton ML. 2003. Evolution in stressful environments II: Adaptive value and costs of plasticity in response to low light in Sinapis arvensis. J Evol Biol 16: $313-323$.

Strange A, Li P, Lister C, Anderson J, Warthmann N, Shindo C, Irwin J, Nordborg M, Dean C. 2011. Major-effect alleles at relatively few loci underlie distinct vernalization and flowering variation in Arabidopsis accessions. PLoS One 6: e19949.

Suarez-Lopez P, Wheatley K, Robson F, Onouchi H, Valverde F, Coupland G. 2001. CONSTANS mediates between the circadian clock and the control of flowering in Arabidopsis. Nature 410: $1116-1120$.

Sultan SE. 2000. Phenotypic plasticity for plant development, function and life history. Trends Plant Sci 5: 537-542.

Sultan SE. 2003. Phenotypic plasticity in plants: A case study in ecological development. Evol Dev 5: 25-33.

Valladares F, Gianoli E, Gómez JM. 2007. Ecological limits to plant phenotypic plasticity. New Phytol 176: 749-763.

Valverde F, Mouradov A, Soppe W, Ravenscroft D, Samach A, Coupland G. 2004. Photoreceptor regulation of CONSTANS protein in photoperiodic flowering. Science 303: 1003-1006.

Van Dyken JD, Wade MJ. 2010. The genetic signature of conditional expression. Genetics 184: 557-570.

Van Kleunen M, Fischer M. 2005. Constraints on the evolution of adaptive phenotypic plasticity in plants. New Phytol 166: 49-60.

Via S. 1993. Adaptive phenotypic plasticity: Target or by-product of selection in a variable environment? Am Nat 142: $352-365$.

Via S, Lande R. 1985. Genotype-environment interaction and the evolution of phenotypic plasticity. Evolution 39: 505522.

Wang JW, Czech B, Weigel D. 2009. MiR156-regulated SPL transcription factors define an endogenous flowering pathway in Arabidopsis thaliana. Cell 138: 738-749.

Weiner J. 2004. Allocation, plasticity and allometry in plants. Perspect Plant Ecol Evol Syst 6: 207-215.

Weinig C, Delph LF. 2001. Phenotypic plasticity early in life constrains developmental responses later. Evolution 55: $930-$ 936.

Wigge PA, Kim MC, Jaeger KE, Busch W, Schmid M, Lohmann JU, Weigel D. 2005. Integration of spatial and temporal information during floral induction in Arabidopsis. Science 309: $1056-1059$.

Wu G, Park MY, Conway SR, Wang JW, Weigel D, Poethig RS. 2009. The sequential action of miR156 and miR172 regulates developmental timing in Arabidopsis. Cell 138: 750-759.

Wund MA. 2012. Assessing the impacts of phenotypic plasticity on evolution. Integr Comp Biol 52: 5-15.

Yamaguchi A, Wu MF, Yang L, Wu G, Poethig RS, Wagner D. 2009. The microRNA-regulated SBP-Box transcription factor 
SPL3 is a direct upstream activator of LEAFY, FRUITFULL, and APETALA1. Dev Cell 17: 268-278.

Yanovsky MJ, Kay SA. 2002. Molecular basis of seasonal time measurement in Arabidopsis. Nature 419: 308-312.

Yoo SK, Chung KS, Kim J, Lee JH, Hong SM, Yoo SJ, Yoo SY, Lee JS, Ahn JH. 2005. CONSTANS activates
SUPPRESSOR OF OVEREXPRESSION OF CONSTANS 1 through FLOWERING LOCUS T to promote flowering in Arabidopsis. Plant Physiol 139: 770-778.

Zhang J, Lechowicz MJ. 1994. Correlation between time of flowering and phenotypic plasticity in Arabidopsis thaliana (Brassicaceae). Am J Bot 81: 1336-1342. 


\section{$\$_{\text {CSH\& }}^{\infty}$ Cold Spring Harbor Symposia SYMPOSIA}

\section{Developmental Plasticity in Plants}

M. de Jong and O. Leyser

Cold Spring Harb Symp Quant Biol 2012 77: 63-73 originally published online December 18, 2012

Access the most recent version at doi:10.1101/sqb.2012.77.014720

References This article cites 103 articles, 22 of which can be accessed free at: http://symposium.cshlp.org/content/77/63.full.html\#ref-list-1

\section{License}

Email Alerting

Receive free email alerts when new articles cite this article - sign up in

Service the box at the top right corner of the article or click here. 